\title{
Correspondence
}

\section{On the proper sterile technique for han- dling of propofol}

To the Editor:

I read with interest the report by Lorenz et al. regarding the proper handling of propofol. ${ }^{1}$ The authors compared two methods of dispensing this medication, either employing a technique of apparently high sterility or what they term a routine method. The result was that no difference in the contamination rate was found. However, a high rate of contamination was reported in both groups.

I was surprised to read that the authors' routine precautions included the re-use of syringes. The reason is that when a syringe has been filled with a medication, the plunger has been pulled out of the syringe's barrel. The plunger may no longer be sterile since contact with the clinician's fingers is likely. Then, when the drug is administered and the plunger is pushed into the barrel, the barrel too will no longer sterile. Consequently, upon refilling the syringe, the drug used may be contaminated. For this reason, syringes should never be refilled. This applies to all medications, not just propofol. In addition, the use of a stopcock when handling propofol is to be condemned. This is because the manipulation of the stopcock is likely to lead to the drug's contamination by the clinician's fingers, especially if some component attached to the stopcock, in this case the propofol syringe, is removed.

Also, this study was not conducted in a blinded manner. Its result was negative, that is the null hypothesis, that the two techniques result in the same frequency of contamination was not disproved. This leads me to wonder if a larger study would reject the null hypothesis. Furthermore, the high frequency of contamination suggests that less than meticulous sterile technique was employed. In particular, the sterile technique that the authors term routine cannot be condoned.

Mitchel B. Sosis MD

Hamilton Square, New Jersey

\section{Reference}

1 Lorenz IH, Kolbitsch C, Lass-Flörl C, et al. Routine handling of propofol prevents contamination as effectively as does strict adherence to the manufacturer's recommendations. Can J Anesth 2002; 49: 347-52.

\section{The lightwand: a useful aid in the dif- ficult tracheostomy}

To the Editor:

Although open, percutaneous and translaryngeal tracheostomy techniques are available, open tracheostomy $(\mathrm{OT})$ remains the most familiar technique at many institutions and still maintains its relevance, particularly where new methods are contraindicated. ${ }^{1}$ It is usually considered a safe procedure with a complication rate ranging from $6 \%$ to $66 \% .^{2}$ This percentage could be higher in the following conditions: morbid obesity, a short and "thick" neck and cervical stiffness. ${ }^{3}$ In these patients and in any case of difficulty in performing OT, we suggest that the Trachlight (TL; Trachlight ${ }^{\mathrm{TM}}$, Laerdal Medical Corp., NY, USA $)^{4}$ could be helpful. We successfully used the TL during a difficult OT in a patient affected by respiratory distress operated under local anesthesia. Because of the short and thick neck and cervical stiffness, it was very difficult for the surgeon to identify the trachea. During the procedure tracheal intubation was needed. Under TL guidance, tracheostomy was carried out easily, without complications.

Light-guided tracheostomy (LT) could minimize the risk of damaging the endotracheal tube or the cuff. ${ }^{5}$ We believe that LT may help tracheal identification in patients with difficult surgical anatomy, a useful and quick procedure to assist surgeons.

In summary, we think the lightwand may be useful, especially in selected difficult cases. We are carrying out a clinical trial in order to evaluate and compare LT and standard open tracheostomy.

Felice Agrò MD

Fabrizio Salvinelli MD

Stefano Gherardi MD

Manuele Casale MD

Rome, Italy

\section{References}

1 Byhahn C, Lischke V, Westphal K. Translaryngeal tracheostomy in highly unstable patients. Anaesthesia 2000; 55: 678-82

2 Friedman $\Upsilon$, Fildes J, Mizock B, et al. Comparison of percutaneous and surgical tracheostomies. Chest 1996; 110: 480-5. 